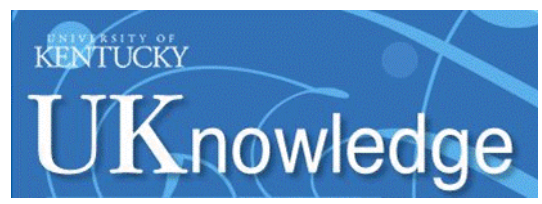

University of Kentucky

UKnowledge

$1-2018$

\title{
Local Loss and Spatial Homogenization of Plant Diversity Reduce Ecosystem Multifunctionality
}

\author{
Yann Hautier \\ Utrecht University, The Netherlands \\ Forest Isbell \\ University of Minnesota - Twin Cities \\ Elizabeth T. Borer \\ University of Minnesota - Twin Cities \\ Eric W. Seabloom \\ University of Minnesota - Twin Cities \\ W. Stanley Harpole

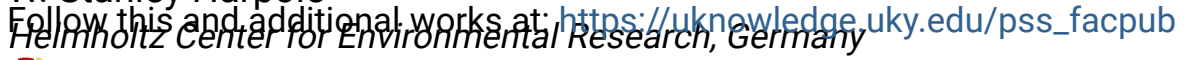 \\ Part of the Ecology and Evolutionary Biology Commons, Environmental Sciences Commons, Plant \\ Sciences Commons, and the Soil Science Commons \\ See next page for additional authors \\ Right click to open a feedback form in a new tab to let us know how this document benefits you.
}

\section{Repository Citation}

Hautier, Yann; Isbell, Forest; Borer, Elizabeth T.; Seabloom, Eric W.; Harpole, W. Stanley; Lind, Eric M.; MacDougall, Andrew S.; Stevens, Carly J.; Adler, Peter B.; Alberti, Juan; Bakker, Jonathan D.; Brudvig, Lars A.; Buckley, Yvonne M.; Cadotte, Marc; Caldeira, Maria C.; Chaneton, Enrique J.; Chu, Chengjin; Daleo, Pedro; Dickman, Christopher R.; Dwyer, John M.; Eskelinen, Anu; Fay, Philip A; Firn, Jennifer; Hagenah, Nicole; Hillebrand, Helmut; Iribarne, Oscar; Kirkman, Kevin P.; Knops, Johannes M. H.; La Pierre, Kimberly J.; and McCulley, Rebecca L., "Local Loss and Spatial Homogenization of Plant Diversity Reduce Ecosystem Multifunctionality" (2018). Plant and Soil Sciences Faculty Publications. 127. https://uknowledge.uky.edu/pss_facpub/127

This Article is brought to you for free and open access by the Plant and Soil Sciences at UKnowledge. It has been accepted for inclusion in Plant and Soil Sciences Faculty Publications by an authorized administrator of UKnowledge. For more information, please contact UKnowledge@lsv.uky.edu. 


\title{
Local Loss and Spatial Homogenization of Plant Diversity Reduce Ecosystem Multifunctionality
}

\author{
Digital Object Identifier (DOI) \\ https://doi.org/10.1038/s41559-017-0395-0
}

Notes/Citation Information

Published in Nature Ecology \& Evolution, v. 2, issue 1, p. 50--56.

(C) 2017 Macmillan Publishers Limited, part of Springer Nature. All rights reserved.

The copyright holder has granted the permission for posting the article here.

This is a post-peer-review, pre-copyedit version of an article published in Nature Ecology \& Evolution. The final authenticated version is available online at: https://doi.org/10.1038/s41559-017-0395-0.

Due to the large number of authors, only the first 30 and the authors affiliated with the University of Kentucky are listed in the author section above. For the complete list of authors, please download this article or visit: https://doi.org/10.1038/s41559-017-0395-0

\section{Authors}

Yann Hautier, Forest Isbell, Elizabeth T. Borer, Eric W. Seabloom, W. Stanley Harpole, Eric M. Lind, Andrew S. MacDougall, Carly J. Stevens, Peter B. Adler, Juan Alberti, Jonathan D. Bakker, Lars A. Brudvig, Yvonne M. Buckley, Marc Cadotte, Maria C. Caldeira, Enrique J. Chaneton, Chengjin Chu, Pedro Daleo, Christopher R. Dickman, John M. Dwyer, Anu Eskelinen, Philip A Fay, Jennifer Firn, Nicole Hagenah, Helmut Hillebrand, Oscar Iribarne, Kevin P. Kirkman, Johannes M. H. Knops, Kimberly J. La Pierre, and Rebecca L. McCulley

This article is available at UKnowledge: https://uknowledge.uky.edu/pss_facpub/127 
Title: Local loss and spatial homogenization of plant diversity reduce ecosystem

\section{multifunctionality}

(1)

Yann Hautier ${ }^{1 *} \underline{0000-0003-4347-7741}$, Forest Isbell ${ }^{2}$, Elizabeth T. Borer ${ }^{2}$, Eric W. Seabloom², W.

Stanley Harpole ${ }^{3,4,5}$, Eric M. Lind ${ }^{2}$, Andrew S. MacDougall ${ }^{6}$, Carly J. Stevens ${ }^{7}$, Peter B. Adler ${ }^{8}$, Juan Alberti $^{9}$, Jonathan D. Bakker ${ }^{10}$, Lars A. Brudvig ${ }^{11}$, Yvonne M. Buckley ${ }^{12}$, Marc Cadotte ${ }^{13}$, Maria C. Caldeira ${ }^{14}$, Enrique J. Chaneton ${ }^{15}$, Chengjin Chu ${ }^{16}$, Pedro Daleo ${ }^{9}$, Christopher R. Dickman $^{17}$, John M. Dwyer ${ }^{18}$, Anu Eskelinen ${ }^{3,4,19}$, Philip A. Fay ${ }^{20}$, Jennifer Firn ${ }^{21}$, Nicole Hagenah $^{22,23}$, Helmut Hillebrand ${ }^{24}$, Oscar Iribarne ${ }^{25}$, Kevin P. Kirkman ${ }^{22}$, Johannes M. H. $\mathrm{Knops}^{26}$, Kimberly J. La Pierre ${ }^{27}$, Rebecca L. McCulley ${ }^{28}$, John W. Morgan ${ }^{29}$, Meelis Pärtel ${ }^{30}$, Jesus Pascual ${ }^{9}$, Jodi N. Price ${ }^{31}$, Suzanne M. Prober ${ }^{32}$, Anita C. Risch ${ }^{33}$, Mahesh Sankaran ${ }^{34,35}$, Martin Schuetz ${ }^{36}$, Rachel J. Standish ${ }^{37}$, Risto Virtanen ${ }^{3,4,19}$, Glenda M. Wardle ${ }^{38}$, Laura Yahdjian $^{15}$, Andy Hector ${ }^{39}$

\footnotetext{
${ }^{1}$ Ecology and Biodiversity Group, Department of Biology, Utrecht University, Padualaan 8, 3584 CH Utrecht, Netherlands.
}

${ }^{2}$ Department of Ecology, Evolution, and Behavior, University of Minnesota, St. Paul, MN, USA.

${ }^{3}$ Department of Physiological Diversity, Helmholtz Center for Environmental Research - UFZ, Permoserstrasse 15, Leipzig 04318, Germany.

${ }^{4}$ German Centre for Integrative Biodiversity Research (iDiv) Halle-Jena-Leipzig, Deutscher Platz 5e, Leipzig 04103, Germany.

${ }^{5}$ Institute of Biology, Martin Luther University Halle-Wittenberg, Am Kirchtor 1, Halle (Saale) 06108, Germany.

${ }^{6}$ Department of Integrative Biology, University of Guelph, Guelph, Ontario Canada N1G 2W1.

${ }^{7}$ Lancaster Environment Centre, Lancaster University, Lancaster, LA1 4YQ, UK. 
${ }^{8}$ Department of Wildland Resources and the Ecology Center, Utah State University, Logan, UT 84322, USA.

${ }^{9}$ Instituto de Investigaciones Marinas y Costeras, UNMdP, CONICET, 7600 Mar del Plata, Argentina.

${ }^{10}$ School of Environmental and Forest Sciences, University of Washington, Box 354115, Seattle, WA 98195-4115, USA.

${ }^{11}$ Department of Plant Biology and Program in Ecology, Evolutionary Biology and Behavior, Michigan State University, East Lansing, MI 48824, USA.

${ }^{12}$ School of Natural Sciences, Zoology, Trinity College Dublin, Dublin 2, Ireland.

${ }^{13}$ University of Toronto Scarborough, Toronto, Ontario, Canada M1C 1A4.

${ }^{14}$ Forest Research Center, ISA, University of Lisbon, 1349-017Lisbon, Portugal.

${ }^{15}$ IFEVA-CONICET, Facultad de Agronomia, Universidad de Buenos Aires, Av. San Martin 4453, 1417 Buenos Aires, Argentina.

${ }^{16}$ State Key Laboratory of Grassland and Agro-Ecosystems, Research Station of Alpine Meadow and Wetland Ecosystems, School of Life Sciences, Lanzhou University, Lanzhou 730000, China.

${ }^{17}$ Desert Ecology Research Group, School of Life and Environmental Sciences, The University of Sydney, NSW 2006, Australia.

${ }^{18}$ School of Biological Sciences, The University of Queensland, St Lucia, QLD 4072, Australia \& CSIRO Land \& Water, EcoSciences Precinct, Dutton Park, QLD 4102, Australia.

${ }^{19}$ Department of Ecology, University of Oulu, FI-90014 University of Oulu, Finland.

${ }^{20}$ USDA-ARS, Grassland Soil and Water Lab, 808 E Blackland Road, Temple, TX, 76502, USA.

${ }^{21}$ School of Biological Sciences, Queensland University of Technology, Brisbane, Australia.

${ }^{22}$ School of Life Sciences, University of KwaZulu-Natal, Pietermaritzburg, South Africa.

${ }^{23}$ South African Environmental Network (SAEON), Grasslands, Forests and Wetlands Node, Queen Elizabeth Park, 1 Peter Brown Drive, Pietermaritzburg, South Africa

${ }^{24}$ Institute for Chemistry and Biology of the marine Environment, University Oldenburg, Germany. 
${ }^{25}$ Instituto de Investigaciones Marinas y Costeras, UNMdP, CONICET, 7600 Mar del Plata, Argentina.

${ }^{26}$ School of Biological Sciences, University of Nebraska - Lincoln, USA.

${ }^{27}$ Smithsonian Environmental Research Center, 647 Contees Wharf Road, Edgewater, Maryland, USA.

${ }^{28}$ Department of Plant \& Soil Sciences, University of Kentucky, Lexington KY 40546-0091, USA.

${ }^{29}$ Department of Ecology, Environment and Evolution, La Trobe University, Bundoora 3083, Victoria, Australia.

${ }^{30}$ Institute of Ecology and Earth Sciences, University of Tartu, Lai 40, Tartu 51005, Estonia.

${ }^{31}$ Institute of Land, Water and Society, Charles Sturt University, Albury, NSW, 2640, Australia.

${ }^{32}$ CSIRO Land and Water, Private Bag 5, Wembley, WA 6913, Australia.

${ }^{33}$ Swiss Federal Institute of Forest, Snow and Landscape Research, 8903 Birmensdorf, Switzerland.

${ }^{34}$ School of Biology, University of Leeds, Leeds LS2 9JT, UK.

${ }^{35}$ National Centre for Biological Sciences, GKVK Campus, Bangalore 560065, India.

${ }^{36}$ Swiss Federal Institute of Forest, Snow and Landscape Research, 8903 Birmensdorf, Switzerland.

${ }^{37}$ School of Veterinary and Life Sciences, Murdoch University, 90 South Street, Murdoch Western Australia 6150.

${ }^{38}$ Desert Ecology Research Group, School of Life and Environmental Sciences, The University of Sydney, NSW 2006, Australia.

${ }^{39}$ Department of Plant Sciences, University of Oxford, Oxford OX1 3RB, UK.

*corresponding author: y.hautier@uu.nl

\section{Abstract:}

Biodiversity is declining in many local communities while also becoming increasingly homogenized across space. Experiments show that local plant species loss reduces ecosystem functioning and services, but the role of spatial homogenization of community composition and the potential interaction between diversity at different scales in 
maintaining ecosystem functioning remains unclear, especially when many functions are considered (ecosystem multifunctionality). We present an analysis of eight ecosystem functions measured in 65 grasslands worldwide. We find that more diverse grasslands those with both species-rich local communities (alpha diversity) and large compositional differences among localities (beta diversity) - had higher levels of multifunctionality. Moreover, alpha and beta diversity synergistically affected multifunctionality, with higher levels of diversity at one scale amplifying the contribution to ecological functions at the other scale. The identity of species influencing ecosystem functioning differed among functions and across local communities, explaining why more diverse grasslands maintained greater functionality when more functions and localities were considered. These results were robust to variation in environmental drivers. Our findings reveal that plant diversity, at both local and landscape scales, contributes to the maintenance of multiple ecosystem services provided by grasslands. Preserving ecosystem functioning therefore requires conservation of biodiversity both within and among ecological communities.

\section{Introduction:}

There is consensus from experiments that higher numbers of plant species at small scales $(\alpha$ diversity) contributes to higher levels of ecosystem functioning ${ }^{1-6}$. However, it remains unclear whether the variation in communities observed across landscapes ( $\beta$ diversity) and the interplay between diversity at local and landscape scales also contributes to the functioning of real-world ecosystems such as natural and semi-natural grasslands ${ }^{7,8}$. This is of particular concern given that large-scale variation in communities is being removed through local species loss $s^{9,10}$ and 
immigration or widespread species replacements leading to homogenization ${ }^{11-13}$. Furthermore, given that ecosystems are managed for multiple functions simultaneously (multifunctionality), and that conservation and management actions are usually implemented across different scales ${ }^{14}$, understanding how plant diversity contributes to maintaining multiple functions is needed from small to larger spatial scales ${ }^{15}$.

Spatial heterogeneity of community composition might contribute to ecosystem multifunctionality through two main mechanisms. First, dissimilarity in functionally important species can maintain functioning across landscapes if different species contribute to different functions in different locations ${ }^{7,8,16,17}$. Second, dissimilarity in species composition among local communities can influence ecological interactions including the movement of organisms and resources important for ecosystem functioning. For example, a local community providing habitat for insect species might provide pollination and pest control to neighbouring communities, thereby contributing to ecosystem functioning at both local and landscape scales ${ }^{18}$. Although a couple of studies have shown that plant diversity contributes to ecosystem multifunctionality at larger spatial scales, they were restricted to artificially constructed landscapes based on simulations within a single experiment in a grassland ${ }^{8}$ or within a panEuropean study in forested ecosystems ${ }^{7}$. Thus, it remains unknown whether multifunctionality relates to biodiversity at larger spatial scales in real-world ecosystems composed of interconnected local communities.

Here, we assess the relationship between plant diversity and ecosystem multifunctionality at local $\left(1 \mathrm{~m}^{2}\right)$ and larger ( $>320 \mathrm{~m}^{2}$, hereafter termed 'landscape') scales using small local plots 
and larger spatial blocks (landscapes composed of interconnected local plots) within 65 grassland sites on five continents, from the Nutrient Network collaborative experiment ${ }^{19}$ (Supplementary Fig. 1, Supplementary Table 1). At each site, we sampled naturally occurring plant diversity and measured ecosystem multifunctionality using eight ecosystem processes and properties ${ }^{3,17}$ (hereafter functions): aboveground live biomass, resource capture aboveground (light interception), resource pools belowground (\% total soil nitrogen and extractable soil phosphorus and potassium), soil carbon storage (\% total soil carbon), litter decomposition and invasion resistance (Methods, Supplementary Table 2). We use the term 'functions' in the broad sense to refer to ecosystem processes and properties, including pools and fluxes of matter and energy $y^{3,16,17,20}$. Measurements were taken in $1 \mathrm{~m}^{2}$ plots grouped into spatial blocks typically spread over $1000 \mathrm{~m}^{2}$ (most sites had three blocks (range: 1 to 6 ) with 10 plots (range: 8 to 12) per study site; Supplementary Table 1).

\section{Results and discussion:}

We first assessed whether local plant species richness, community dissimilarity among local communities, and their interaction were associated with ecosystem multifunctionality. We measured species richness as the average number of plant species per $1 \mathrm{~m}^{2}$ plot within spatial blocks ( $\bar{\alpha}$, average $\alpha$ diversity), and community dissimilarity as the mean pairwise difference in plant species composition among plots within spatial blocks ( $\beta$ diversity). The $\bar{\alpha}$ and $\beta$ diversity explanatory variables are both mathematically independent in principle and statistically independent in practice $(\mathrm{R}=0.076, \mathrm{P}=0.28, \mathrm{~N}=206)$, allowing us to consider their independent and interactive relationships with ecosystem multifunctionality. We quantified ecosystem multifunctionality using two approaches ${ }^{21}$ (Methods). The average multifunctionality $^{22}$ approach, 
which provides a relatively interpretable metric and the multiple-threshold multifunctionality approach $^{23}$ which assesses how many functions reach high levels. We calculated average multifunctionality as the mean of all standardized functions within spatial blocks ${ }^{8}$ and multiplethreshold multifunctionality as the mean number of functions per plot within spatial blocks that exceeded threshold values between 5 and $95 \%$ of the observed maximum value for each function.

We found the interactive effect of local species richness ( $\bar{\alpha}$ diversity) and community dissimilarity ( $\beta$ diversity) to be the strongest contributor to average multifunctionality $\left(\mathrm{F}_{1,202}=\right.$ 8.88, $\mathrm{P}=0.003$, Fig. 1, Supplementary Fig. 2 and 3). Specifically, average multifunctionality and local species richness were positively related at intermediate to high community dissimilarity but unrelated at low dissimilarity (Fig. 1a, Fig. 2a). Similarly, average multifunctionality and community dissimilarity were positively related at high species richness but unrelated at low to intermediate richness (Fig. 1b, Fig. 2a). These interactions were generally consistent throughout habitat types (Fig. 2b). These results indicate that diversity at the local $(\bar{\alpha})$ and landscape $(\beta)$ scale may synergistically affect multifunctionality, with higher levels of diversity at one scale amplifying the contribution to ecological functions at the other scale. This also suggests that losing diversity at one scale may have cascading effects on the other scale by weakening its potential to maintain high ecological functioning. In other words, the homogenization of biotic communities could increase the effect of local species loss on ecosystem functioning. Our results were independent of the multifunctionality measure chosen; results of our analyses using multiple-threshold multifunctionality did not differ qualitatively from the results using average multifunctionality (Supplementary Fig. 4). Future studies could more completely consider 
measuring all ecosystem functions related to realistic management objectives and address scenarios representing different management objectives by calculating multifunctionality metrics with different weighing for each ecosystem functions.

Synergistic effects of $\bar{\alpha}$ and $\beta$ diversity were similar regardless of whether functions were considered separately or together (Supplementary Fig. 2). However, in terms of relative contribution to explained variation, some ecosystem functions depended mostly on $\bar{\alpha}$ diversity, whereas others depended mostly on $\beta$ diversity (Supplementary Fig. 3 and 5, Supplementary Table 3). Synergistic effects contributed the most to aboveground live biomass and litter decomposition, $\bar{\alpha}$ diversity to soil potassium and invasion resistance and $\beta$ diversity to light interception, soil carbon, soil nitrogen, and soil phosphorus. These results suggest that high levels of diversity at any single scale may not maintain all functions at desirable levels, but instead that high levels of diversity at multiple scales may be required to maintain multiple functions simultaneously.

We used a multi-model inference approach to assess the relative importance of $\bar{\alpha}, \beta$ diversity, their interaction, and key environmental covariates including geographic, climatic and edaphic variables (Methods) on each individual function and on the average multifunctionality. We found that the interactive effect of $\bar{\alpha}$ and $\beta$ diversity was included in the four best and most parsimonious models which explained more than $32 \%$ of the variance in multifunctionality. Relative to other environmental predictors, the interactive effect of $\bar{\alpha}$ and $\beta$ diversity was the third best predictor of multifunctionality after mean temperature during the wettest four months and mean annual precipitation (Supplementary Fig. 6). The importance of the interaction 
between local and landscape scale diversity further manifested through it being a better predictor of multifunctionality than many other environmental predictors, including climatic variables such as mean annual temperature and edaphic variables such as soil $\mathrm{pH}$.

Higher multifunctionality was associated with warmer temperatures during the wettest four months, larger variation in temperature and higher precipitation (Supplementary Table 4). The relationship between plant diversity and average multifunctionality was generally robust across environmental gradients. The slope of the relationship between $\bar{\alpha}$ diversity and multifunctionality did not vary with our environmental predictors while $\beta$ diversity effects on multifunctionality increased with increasing soil silt and clay content (likely indicators of soil fertility) and decreased with increasing variation in both temperature and total soil nitrogen (Supplementary Table 5).

Similar to the multifunctionality analysis, the best and most parsimonious model describing individual functions included plant diversity ( $\bar{\alpha}$ and/or $\beta$ and/or the interaction) (Supplementary Table 4), and a subset of environmental variables were better predictors of individual functions relative to plant diversity (Supplementary Fig. 6). Plant diversity contributed less to invasion resistance compared to other environmental factors. Effects of environmental variables on individual functions included an association of warmer temperatures with lower plant biomass, percent total soil carbon, and invasion resistance and higher light interception, percent total soil nitrogen and extractable soil potassium. Similarly, higher precipitation was associated with higher plant biomass, light interception, percent total soil carbon and invasion resistance and 
lower percent total soil nitrogen, extractable soil phosphorus, extractable soil potassium and litter decomposition (Supplementary Table 4).

Next, we assessed whether ecological interactions between interconnected communities contribute to the positive relationship between plant diversity and ecosystem multifunctionality. To do so, we compared the results from our observed landscapes composed of interconnected local plots within blocks with results of artificially constructed landscapes simulating reduced interconnection between local communities. Each simulated landscape was composed of ten plots randomly drawn from local plots belonging either to different blocks within sites (average interconnection) or to different sites within habitat type (low interconnection); and from which $\bar{\alpha}$ and $\beta$ diversity and average multifunctionality were calculated. In our simulated landscapes, local species richness ( $\bar{\alpha}$ diversity) and community dissimilarity ( $\beta$ diversity) interacted to affect the average multifunctionality (simulated landscapes within sites $\mathrm{F}_{1,6496}=225.26, \mathrm{P}<0.001$, $\mathrm{N}=6500$, simulated landscapes within habitats $\mathrm{F}_{1,4996}=30.43, \mathrm{P}<0.001, \mathrm{~N}=5000$ ). When compared to our observed landscapes (Fig. 2a and b), artificially reducing interconnection between communities either within sites (Fig. 2c) or within habitats (Fig. 2d) did not influence the relationships of $\bar{\alpha}$ and $\beta$ diversity with average multifunctionality. Similar to our observed landscapes, simulated landscapes generally showed stronger association between species richness and average multifunctionality at high community dissimilarity and between community dissimilarity and average multifunctionality at high species richness.

Finally, we assessed whether dissimilarity in functionally important species contribute to ecosystem multifunctionality. We identified the sets of species most important for maintaining 
ecosystem functioning for each function in each locality (spatial block) at each site using three analytical approaches that range in how conservative they are in identifying species effects (Methods): stepwise backward-deletion multiple regression ${ }^{16,17}$, randomization ${ }^{24}$, and multimodel inference ${ }^{25}$. For each approach, we quantified the degree of functional and spatial overlap between species sets ${ }^{16,17}$. For example, we quantified functional overlap between all pairs of functions within spatial blocks. Functional overlap values of one or zero would indicate respectively that completely identical or completely unique sets of species were important for maintaining different functions in any particular spatial block. Finally, for each site, we quantified the proportion of unique species that maintained ecosystem functioning at least once across all combinations of functions for each spatial block and across all combinations of spatial blocks for each function considered.

We found low functional and spatial overlap in the sets of species influencing ecosystem functions (Supplementary Fig. 7). Thus, the identity of the species most important for maintaining ecosystem functioning differed between ecosystem functions and among local communities, resulting in a higher proportion of species required for maintaining ecosystem functioning when more functions (Fig. 3a) or localities (spatial blocks, Fig. 3b) were independently considered ${ }^{16}$; and explaining why greater overall ecosystem functioning was found to be associated with greater local plant species and greater spatial heterogeneity in community composition (Fig. 1). These positive associations between the proportion of species maintaining functioning and the range of functions or localities considered were observed for each of the three approaches investigated (Fig. 3). For example, predictions from the most to the least conservative method show that between 10 and $28 \%$ of the species pool maintained one 
function in one block, while between 19 and 37\% maintained the same function in three blocks, and between 39 and 54\% maintained the same function in six blocks simultaneously (Fig. 3b). This suggests that while estimates of the number of species important for maintaining functioning may vary with analytical approach, the qualitative results are robust to methodology. Analyses using presence-absence instead of percent species cover, or using only sites with three or fewer spatial blocks, yielded qualitatively similar results (Supplementary Fig. 7). Our results indicate that no single plant species maintains all ecosystem functions in all locations, but rather that more species and greater heterogeneity in species composition across the landscape both contribute to and enhance ecosystem multifunctionality (Supplementary Fig. 8). Together, these analyses suggest that the effects of diversity on multifunctionality are mainly due to species traits and how these traits interact with local environmental conditions and do not point to any additional effects of ecological interactions between interconnected communities.

Our results, based on standardized data collected from grasslands around the world, provide robust, general evidence that plant diversity at the local and landscape scale is associated with more reliable functioning of grassland ecosystems and contribute to the increasing body of knowledge cautioning about the functional consequences of local species loss and biotic homogenization $^{7,8,11,16,17,20,22,26-28}$. Consequently, human activities that simplify ecosystems through the loss of plant diversity ${ }^{9,11-13}$ are likely to diminish the capacity of natural systems to supply essential ecosystem functions, while the maintenance and restoration of plant diversity at local and landscape scales should help ensure the reliable provision of ecosystem services.

\section{Methods:}


The Nutrient Network experiment. The 65 study sites are part of the Nutrient Network Global

Research Cooperative (NutNet, Supplementary Fig. 1, Supplementary Table 1, http://nutnet.org $/)^{19}$. Detailed description of site selection, methods and measurements are available in Borer et al. ${ }^{19}$. Plots at all sites were $5 \times 5 \mathrm{~m}$ (separated by at least $1 \mathrm{~m}$ walkways) spread over an area of at least $1000 \mathrm{~m}^{2}$. Sampling was done in $1 \mathrm{~m}^{2}$ plots grouped into spatial blocks spread over $>320 \mathrm{~m}^{2}$ (typically three blocks (range: 1 to 6 ) of 10 plots (range: 8 to 12 ) per study site; Supplementary Table 1) and followed a standardized protocol at all sites ${ }^{19}$. The analyses presented here include all NutNet sites that contributed to pre-treatment data on community-level functions in all plots and therefore do not include either of the nutrient addition or consumer exclosure treatments. Two sites that contributed data were excluded from these analyses because they did not lay out plots in separate spatial blocks (sevi.us and jorn.us).

Diversity and abundance. A $1 \times 1 \mathrm{~m}$ area within each plot was permanently marked and sampled for species richness during the season of peak biomass. Alpha diversity was the number of plant species per $1 \mathrm{~m}^{2}$ plot and average alpha diversity $(\bar{\alpha})$ the average number of plant species per plot within spatial blocks. Beta diversity ( $\beta$ ) was the dissimilarity in plant species composition among plots within spatial blocks (differences in $1 \mathrm{~m}^{2}$ plots among blocks within each site), which is the complement to Sørensen's similarity index $(o)(\beta=1-o)$ ranging from 0 (completely similar, homogeneous) to 1 (completely dissimilar, heterogeneous). Percent cover was estimated independently for each species, so that total summed cover can exceed $100 \%$ for multilayer canopies. 
Ecosystem functions and properties. Aboveground live biomass $\left(\mathrm{g} \mathrm{m}^{-2}\right)$ was estimated destructively at growing season peak by clipping at ground level all aboveground biomass of individual plants rooted within two $0.1 \mathrm{~m}^{2}(10 \times 100 \mathrm{~cm})$ strips immediately adjacent to the permanent $1 \times 1 \mathrm{~m}$ subplot. Biomass was sorted into current (live and recently senescent material) and previous year's growth (litter). For shrubs and subshrubs, leaves and current year's woody growth were collected. Biomass was dried at $60^{\circ} \mathrm{C}$ to a constant mass and weighed to the nearest $0.01 \mathrm{~g}$. Resource capture aboveground was measured as photosynthetically active radiation (PAR) at the same time and in the same $1 \times 1 \mathrm{~m}$ plot sample for species richness. Light readings were taken using a $1 \mathrm{~m}$ PAR sensor (e.g., Decagon, Apogee) on a cloudless day as close to solar noon as possible (i.e. 11 am to $2 \mathrm{pm}$ ). For each plot, we took two light measurements at ground level (at opposite corners of the $1 \times 1 \mathrm{~m}$ plot, diagonal to each other) and one above the canopy. The complement to the ratio represents the percentage of light intercepted at the ground (percentage of intercepted PAR). Adjacent to each plot, resource pools belowground were estimated using 250 grams of air-dried soil. Total soil $\% \mathrm{C}$ and $\% \mathrm{~N}$ were measured using dry combustion GC analysis (COSTECH ESC 4010 Element Analyzer) at the University of Nebraska. Extractable soil P and K (p.p.m.) were quantified using the Mehlich-3 extraction method and p.p.m. concentration was estimated using ICP (A\&L Analytical Laboratory, Memphis, TN, USA). Litter turnover $\left(\mathrm{y}^{-1}\right)(\mathrm{k})$ as a proxy for litter decomposition was estimated using an equation derived from Olson ${ }^{29,30}$ for deciduous forest decay rates:

$$
k=-\log \left(1-\left(\frac{\text { live biomass }}{\text { total biomass }}\right)\right)
$$

where live biomass is the standing stock during peak season and total biomass is live biomass plus litter collected at the same time ${ }^{30}$. Although our experimental system is not a forested system as modeled in Olson's paper, both are deciduous with annual biomass contributions to the 
litter pool. Native dominance as a proxy for invasion resistance was estimated as the ratio of native to invasive species cover. Note that some sites measured only a subset of these eight functions (Supplementary Table 1). In the calculation of multifunctionality, we used the inverse of soil N, P and $\mathrm{K}$ as lower levels of unconsumed resources are consistent with higher uptake and lower potential for leaching.

Trade-offs between functions. To investigate potential trade-offs between individual functions, we calculated Pearson's correlation coefficients between each pair of individual standardized functions. Of the possible 28 combinations of pairs of functions, we found significant positive correlations between eleven pairs and significant negative correlations between five pairs (Supplementary Table 2). We found a strong negative correlation between our inverse measure of percent total $\mathrm{N}$ and percent total $\mathrm{C}(-0.96)$. We kept both variables in our analyses because a negative correlation meant that choosing one function or the other would favour either a positive or negative impact of diversity on average multifunctionality. In contrast, retaining both variables demonstrates a trade-off between them. Moreover, our results were qualitatively similar when we used either percent total $\mathrm{N}$ or the soil C:N ratio. All the other correlations were lower than 0.30 .

\section{Community-level analyses.}

Ecosystem multifunctionality. We quantified ecosystem multifunctionality in whole communities of interacting species using two methods ${ }^{21}$ : the average and multiple threshold approaches.

We standardized each function by the maximum observed value across all sites to remove the effects of differences in measurement scale between functions ${ }^{21}$. We then calculated block 
average multifunctionality as the mean of all standardized functions within spatial blocks ${ }^{8}$. The average multifunctionality metric is intuitive and easy to interpret, but it does not incorporate potential tradeoffs between functions that perform at high levels when others perform at low levels.

The multiple threshold approach ${ }^{8,23,31,32}$ overcomes this limitation and tests whether diversity is associated with higher numbers of functions exceeding discrete threshold values considered to be minimal for desirable ecosystem functioning. We calculated the number of functions per plot that exceeded a given threshold value, expressed as a percentage of each maximum function value. Here, we defined maximum level of functioning for each function as the average of the top four values for each function across all sites. We then calculated multiple-threshold multifunctionality ${ }^{23}$ as the mean number of functions that exceeded a given threshold within spatial blocks. In practice, a range of thresholds is usually explored. We calculated the average number of functions exceeding functional thresholds between 5 and $95 \%$ of this maximum per plot. Thus, for each block, 91 values (counts of functions) were generated, one for each discrete threshold value between 5 and $95 \%$.

Association between plant diversity and ecosystem functioning (average multifunctionality). We explored the direct relationships of plant diversity, measured as the average species richness $(\bar{\alpha})$, community dissimilarity $(\beta)$ and their interaction $(\bar{\alpha}: \beta)$, with each individual standardized function and the average multifunctionality across the 65 sites (Fig. 1, Fig. 2a) and within habitat types (Fig. 2b) using generalized linear models (GLMs) with a quasibinomial error distribution and logit link function. See the section hereafter 'Assessing whether ecological interactions between interconnected communities contribute to ecosystem multifunctionality' for a 
description of how the habitat types were selected. In order to visualize the interactive effect of $\bar{\alpha}$ and $\beta$ diversity on average multifunctionality, we divided the data set into three equal groups corresponding to low (Low), intermediate (Int) and high (High) levels of $\bar{\alpha}$ or $\beta$ diversity and fitted separate models for each group. This means that we fitted relationships between $\bar{\alpha}$ diversity and average multifunctionality at low, intermediate and high levels of $\beta$ diversity. Similarly, we fitted relationships between $\beta$ diversity and average multifunctionality at low, intermediate and high levels of $\bar{\alpha}$ diversity. Due to similar fit we subsequently grouped the intermediate and high levels (Int-High) of $\bar{\alpha}$ diversity and the low and intermediate levels (Low-Int) of $\beta$ diversity (Fig. 1, Supplementary Fig. 5). We also assessed the relative contribution of $\bar{\alpha}, \beta$ diversity and $\bar{\alpha}: \beta$ to average multifunctionality by using multivariate models to calculate standardized regression coefficients (Supplementary Fig. 2) and the percentage of variance explained (percent of $\mathrm{R}^{2}$, Supplementary Fig. 3) for each diversity metric.

\section{Association between plant diversity and ecosystem functioning (multiple-threshold}

multifunctionality). To assess the relationship between plant diversity and multiple-threshold multifunctionality, we fitted separate models for each of the 91 discrete threshold values between 5 and $95 \%$, and recorded the slope and associated $95 \%$ confidence intervals (Supplementary Fig. 4). Because the responses in each of the 91 models were integers (counts of functions exceeding the particular threshold) we used GLMs with a quasipoisson error distribution (to account for observed over-dispersion) and identity link function ${ }^{21}$. We rerun the analysis adjusting for the fact that some functions were not measured for all sites by measuring the percentage of measured functions exceeding a given threshold. Because the responses in each of the 91 models were percentages we fitted GLMs with a quasibinomial error distribution and logit link function ${ }^{21}$. 
Results did not qualitatively differ between the two analyses. For both analyses, we included environmental variables because the relationship between plant diversity and multifunctionality may covary with environmental factors correlated to both plant diversity and ecosystem multifunctionality.

Relative importance of plant diversity and environmental predictors. We used a multi-model inference approach based on Akaike information criterion (AIC) and ordinary least square (OLS) regression to assess the relative importance of $\bar{\alpha}, \beta$ diversity and $\bar{\alpha}: \beta$ and key environmental predictors on each individual function and on the average multifunctionality (Supplementary Fig. 6, Supplementary Table 4). We fitted separate models for each function and the average multifunctionality as response variables and fifteen potential environmental predictors including geographic, climatic and edaphic variables. Geographic variables included latitude and longitude. Climatic variables were derived from the WorldClim Global Climate database (version 1.4; http://www.worldclim.org/) ${ }^{33}$. Due to multicollinearity between many of the climatic variables, we first fitted a principal component analysis (PCA) to reduce their number, resulting in a subset of bioclimatic variables representing annual trends (mean annual temperature $\left({ }^{\circ} \mathrm{C}\right)$ and precipitation $(\mathrm{mm})$ ), seasonality (mean annual range in temperature, standard deviation in temperature, coefficient of variation of precipitation) and extreme or limiting environmental factors (mean temperature during the wettest four months) ${ }^{34}$. Edaphic variables included $\mathrm{pH}$, bulk density, soil nutrient heterogeneity (coefficient of variation in total soil nitrogen, extractable soil phosphorus and extractable soil potassium) and soil texture (percent silt, percent clay and percent sand). Again due to multicollinearity between soil texture variables, we used percent silt and percent clay in our analyses. 
Relationship between plant diversity and average multifunctionality across environmental gradients. To assess whether the relationship between plant diversity and average multifunctionality varied across environmental gradients, we first determined the slopes of the relationships of $\bar{\alpha}$ and $\beta$ diversity with average multifunctionality within each site using linear mixed-effects models and site as random effect allowing both the intercepts and slopes of the regression to vary among sites. We then assessed the relationships between the slopes of relationships of $\bar{\alpha}$ and $\beta$ diversity with average multifunctionality as response variable and each environmental variable as explanatory variables (Supplementary Table 5).

\section{Assessing whether ecological interactions between interconnected communities contribute} to ecosystem multifunctionality.

To assess the contribution of ecological interactions to multifunctionality, we constructed artificial landscapes from our grassland plots belonging either to different blocks within sites (average interconnection) or to different sites within habitats (low interconnection); and from which $\bar{\alpha}$ and $\beta$ diversity and average multifunctionality were calculated as described above. Simulated landscapes within sites. Within each site, we constructed 100 artificial landscapes each composed of ten plots randomly selected, without replacement, across the different blocks. With 65 sites, this resulted in 6500 landscapes.

Simulated landscapes within habitats. Within each habitat (Supplementary Table 1), we constructed 1000 artificial landscapes each composed of ten plots randomly selected, without replacement, across the different sites. The number of sites within each habitat was relatively low (ranging between one and eight) and many habitats were represented by only a few sites. In order to ensure that our landscape were composed of unique plot combinations, we selected the 
habitats represented by more than four sites. Due to their similarity, alpine and montane grasslands were subsequently grouped together. This resulted in five habitats with a total of 5000 landscapes.

For each of the observed and simulated landscapes within sites and within habitats, we quantified the standardized regression coefficients of the relationships of plant diversity, measured as the average species richness $(\bar{\alpha})$, community dissimilarity $(\beta)$ and their interaction $(\bar{\alpha}: \beta)$, with average multifunctionality using OLS regression. Again, in order to visualize the interactive effect of $\bar{\alpha}$ and $\beta$ diversity on average multifunctionality, we divided the data set into three equal groups corresponding to low (Low), intermediate (Int) and high (High) levels of $\bar{\alpha}$ or $\beta$ diversity and fitted separate models for each group (Fig. 2).

\section{Species-level analyses: assessing whether dissimilarity in functionally important species} contribute to ecosystem multifunctionality.

Identifying sets of species most important for maintaining ecosystem functioning. We started by identifying the sets of species most important for maintaining ecosystem functioning for each function in each spatial block at each site, based on three approaches proposed in the ecological literature that range in how conservative they are in identifying species effects: stepwise-deletion multiple regression ${ }^{16,17,35}$, randomization ${ }^{24}$ and multimodel inference ${ }^{25}$. For each approach, we modeled ecosystem functioning in response to the abundance (percent cover, Fig. 3) or the presence-absence of each species in each plot (Supplementary Fig. 7). For the presence-absence analysis, some species were present in every plot within spatial blocks and could not be included in the analyses as their contributions could not be statistically estimated. However, all species could be included in analyses using abundance data, as abundance values varied among plots for 
each species. Where the results overlapped with the presence/absence data they were qualitatively similar (Fig. 3, Supplementary Fig. 7).

Stepwise-deletion multiple regression identified the most parsimonious set of species influencing each ecosystem function based on information criteria ${ }^{36}$. We implemented this procedure using the stepAIC function in the MASS library ${ }^{37}$ of $\mathrm{R}^{16,17,35}$. In stepwise-deletion analyses, multiple models can have nearly equivalent support, making it misleading to choose a single best model in that case. Multimodel inference addresses this problem by accounting for model selection uncertainty and reducing model selection bias ${ }^{38}$. In this sense multimodel inference is more robust and conservative than stepwise-deletion. We implemented multimodel inference using the glmulti function in the glmulti R package ${ }^{25}$. While stepwise-deletion and multimodel inference require designs that include each species in a variety of compositional treatments (typical of most but not all biodiversity experiments) ${ }^{21}$, randomization is advocated for observational studies lacking imposed compositional treatments ${ }^{24}$. The effect of each species on each function is measured in multiple plots as the difference between the average of a function in the presence and absence of a particular species. The sets of species that show strong influences on each function are then identified by randomly reassigning the values of the ecosystem function to the different plots for 1000 iterations $^{24}$.

\section{Comparing sets of species most important for maintaining ecosystem functioning. After} identifying the sets of species most important for maintaining ecosystem functioning in each plot, we quantified overlap $o$ between species sets for each of the stepwise-deletion multiple regression, randomization and multimodel inference approaches. To test whether different sets of species maintained ecosystem functioning for different functions in different spatial blocks, we 
quantified functional and spatial overlap between species sets. All comparisons were made within spatial blocks so that differences between pairs of functions or pairs of spatial blocks were not due to sampling from multiple species pools. We quantified functional overlap between functions $a$ and $b$ in a particular spatial block and spatial overlap between spatial blocks $a$ and $b$ for a particular function using Sørensen's similarity index ${ }^{16,17}$ :

$$
o=\frac{\left|E_{a} \cap E_{b}\right|}{0.5\left(\left|E_{a}\right|+\left|E_{b}\right|\right)}
$$

Where $\left|E_{a}\right|$ is the number of species that promoted ecosystem functioning for function or spatial block $a,\left|E_{b}\right|$ is the number of species that promoted ecosystem functioning for function or spatial block $b$ and $\left|E_{a} \cap E_{b}\right|$ is the number of species that promoted ecosystem functioning for both functions or spatial blocks. This allowed us to test whether identical (overlap =1), unique (overlap $=0)$ or somewhat different $(0<$ overlap $<1)$ sets of species promoted ecosystem functioning for different functions at different spatial blocks.

\section{Accumulation of species across functions and spatial blocks. For each approach, we then} assessed how the proportion of species maintaining functioning changed as more functions or spatial blocks were considered. We quantified the accumulation of species that maintained ecosystem functioning across all combinations of functions for each spatial block and across all combinations of spatial blocks for each function considered. For example, to estimate how the proportion of species maintaining functioning changed as more functions were considered, we sampled all combinations of the eight functions (that is, all pairs, groups of three, etc.), and recorded the number of unique species that maintained functioning, the total number of species, for each combination. The proportion of species was then calculated by dividing the number of species that maintained functioning by the total number of species per spatial block. This was 
repeated for each spatial block, at each site. We modelled the relationships between the proportion of species that maintained ecosystem functioning and the number of functions or spatial blocks, for each of the stepwise-deletion multiple regression, randomization tests and multimodel inference approaches, using quasibinomial GLMs including "approaches" as a factor with three levels. The number of spatial blocks per site range between one and six, meaning that the relationship between the proportion of species that maintained ecosystem functioning and the number of spatial blocks could be driven by the few sites with more than three blocks (Fig. 3). We therefore re-run the analyses using a subset of the data including only sites with three or fewer spatial blocks (Supplementary Fig. 7). All analyses were conducted in R 2.15.1 ${ }^{39}$.

Data availability. The datasets generated during and/or analysed during the current study are available from the corresponding author on reasonable request.

\section{Acknowledgments:}

The research leading to these results has received funding from the European Union Seventh Framework Programme (FP7/2007-2013) under grant agreement no. 298935 to Y.H. (with A.H. and E.W.S.). This work was generated using data from the Nutrient Network (http://www.nutnet.org) experiment, funded at the site-scale by individual researchers.

Coordination and data management have been supported by funding from the National Science Foundation Research Coordination Network (NSF-DEB-1042132) to E.T.B. and E.W.S, and from the Long Term Ecological Research (LTER) programme (NSF-DEB-1234162), and the Institute on the Environment at the University of Minnesota (DG-0001-13). We also thank the Minnesota Supercomputer Institute for hosting project data, and the Institute on the Environment 
for hosting Network meetings. We thank R. S. L. Veiga for suggestions that improved the manuscript.

\section{Author Contributions:}

Y.H., F.I. and A.H. developed and framed research questions. Y.H., F.I. and A.H. analysed the data and wrote the paper with contributions and input from all authors. E.T.B., E.W.S., K.L.P., and J.D.B. contributed to data analysis. E.W.S., E.T.B., W.S.H. and E.M.L. are Nutrient Network coordinators. All authors collected data used in this analysis. Author contribution matrix provided as Supplementary Table 6.

\section{Declaration of Financial Competing Interests}

The authors declare no competing financial competing interests.

\section{Additional information}

Supplementary information is available for this paper.

Reprints and permissions information is available at www.nature.com/reprints.

Correspondence and requests for materials should be addressed to Y.H.

How to cite this article: Hautier, Y. et al. Local loss and spatial homogenization of biodiversity reduce ecosystem multifunctionality. Nat. Ecol. Evol. X, xxxx (201x).

Publisher's note: Springer Nature remains neutral with regard to jurisdictional claims in published maps and institutional affiliations. 
5531 Cardinale, B. J. et al. Biodiversity loss and its impact on humanity. Nature 486, 59-67, $554 \quad(2012)$.

555

556

557

2 Naeem, S., Bunker, D. E., Hector, A., Loreau, M. \& Perrings, C. Biodiversity, Ecosystem Functioning, \& Human Wellbeing: An Ecological and Economic Perspective. Vol. 44 (Oxford University Press, 2010).

3 Hooper, D. U. et al. Effects of biodiversity on ecosystem functioning: A consensus of current knowledge. Ecological Monographs 75, 3-35, (2005).

4 Loreau, M. et al. Ecology - Biodiversity and ecosystem functioning: Current knowledge and future challenges. Science 294, 804-808, (2001).

5 Tilman, D., Isbell, F. \& Cowles, J. M. Biodiversity and Ecosystem Functioning. Annual Review of Ecology, Evolution, and Systematics 45, 471-493, (2014).

6 Hautier, Y. et al. Anthropogenic environmental changes affect ecosystem stability via biodiversity. Science 348, 336-340, (2015).

7 van der Plas, F. et al. Biotic homogenization can decrease landscape-scale forest multifunctionality. Proc. Natl. Acad. Sci. U. S. A. 113, 3557-3562, (2016).

8 Pasari, J. R., Levia, T., Zavaletaa, E. S. \& Tilman, D. Several scales of biodiversity affect ecosystem multifunctionality. Proc. Natl. Acad. Sci. U. S. A. 110, 10219-10222, (2013).

9 Newbold, T. et al. Global effects of land use on local terrestrial biodiversity. Nature 520, 45$50,(2015)$.

10 Murphy, G. E. P. \& Romanuk, T. N. A meta-analysis of declines in local species richness from human disturbances. Ecology and Evolution 4, 91-103, (2014). 
11 McKinney, M. L. \& Lockwood, J. L. Biotic homogenization: a few winners replacing many losers in the next mass extinction. Trends in Ecology \& Evolution 14, 450-453, (1999).

12 Vellend, M. et al. Global meta-analysis reveals no net change in local-scale plant biodiversity over time. Proc. Natl. Acad. Sci. U. S. A. 110, 19456-19459, (2013).

13 Dornelas, M. et al. Assemblage time series reveal biodiversity change but not systematic loss. Science 344, 296-299, (2014).

14 Ferrier, S. et al. Mapping more of terrestrial biodiversity for global conservation assessment. Bioscience 54, 1101-1109, (2004).

15 Isbell, F. et al. Linking the influence and dependence of people on biodiversity across scales. Nature 546, 65-72, (2017).

16 Isbell, F. et al. High plant diversity is needed to maintain ecosystem services. Nature 477, 199-202, (2011).

17 Hector, A. \& Bagchi, R. Biodiversity and ecosystem multifunctionality. Nature 448, 188$190,(2007)$.

18 Tscharntke, T., Klein, A. M., Kruess, A., Steffan-Dewenter, I. \& Thies, C. Landscape perspectives on agricultural intensification and biodiversity - ecosystem service management. Ecol. Lett. 8, 857-874, (2005).

19 Borer, E. T. et al. Finding generality in ecology: a model for globally distributed experiments. Methods in Ecology and Evolution 5, 63-73, (2013).

20 Mori, A. S. et al. Low multifunctional redundancy of soil fungal diversity at multiple scales. Ecol. Lett. 19, 249-259, (2016). 
21 Byrnes, J. E. K. et al. Investigating the relationship between biodiversity and ecosystem multifunctionality: challenges and solutions. Methods in Ecology and Evolution 5, 111-124, (2014).

22 Maestre, F. T. et al. Plant Species Richness and Ecosystem Multifunctionality in Global Drylands. Science 335, 214-218, (2012).

23 Zavaleta, E. S., Pasari, J. R., Hulvey, K. B. \& Tilman, G. D. Sustaining multiple ecosystem functions in grassland communities requires higher biodiversity. Proc. Natl. Acad. Sci. U. S. A. 107, 1443-1446, (2010).

24 Gotelli, N. J., Ulrich, W. \& Maestre, F. T. Randomization tests for quantifying species importance to ecosystem function. Methods in Ecology and Evolution 2, 634-642, (2011).

25 Calcagno, V. \& de Mazancourt, C. glmulti: an R package for easy automated model selection with (generalized) linear models. Journal of Statistical Software 34, (2010).

26 Wagg, C., Bender, S. F., Widmer, F. \& van der Heijden, M. G. A. Soil biodiversity and soil community composition determine ecosystem multifunctionality. Proc. Natl. Acad. Sci. U. S. A. 111, 5266-5270, (2014).

27 Delgado-Baquerizo, M. et al. Microbial diversity drives multifunctionality in terrestrial ecosystems. Nature Communications 7, (2016).

28 Jing, X. et al. The links between ecosystem multifunctionality and above- and belowground biodiversity are mediated by climate. Nature Communications 6, (2015).

29 Olson, J. Energy storage and the balance of producers and decomposers in ecological systems. Ecology, 322-331, (1963).

30 O'Halloran, L. R. et al. Regional Contingencies in the Relationship between Aboveground Biomass and Litter in the World's Grasslands. Plos One 8, (2013). 
618

619

620

621

622

623

624

625

626

627

628

629

630

631

632

633

634

635

636

637

638

31 Gamfeldt, L., Hillebrand, H. \& Jonsson, P. R. Multiple functions increase the importance of biodiversity for overall ecosystem functioning. Ecology 89, 1223-1231, (2008).

32 Peter, H. et al. Multifunctionality and Diversity in Bacterial Biofilms. Plos One 6, (2011).

33 Hijmans, R. J., Cameron, S. E., Parra, J. L., Jones, P. G. \& Jarvis, A. Very high resolution interpolated climate surfaces for global land areas. International Journal of Climatology 25, 1965-1978, (2005).

34 Hautier, Y. et al. Eutrophication weakens stabilizing effects of diversity in natural grasslands. Nature 508, 521-525, (2014).

35 He, J. Z., Ge, Y., Xu, Z. H. \& Chen, C. R. Linking soil bacterial diversity to ecosystem multifunctionality using backward-elimination boosted trees analysis. Journal of Soils and Sediments 9, 547-554, (2009).

36 McCullagh, P. \& Nelder, J. A. Generalized Linear Models. Second edn, (Chapman and Hall, 1989).

37 Venables, W. N. \& Ripley, B. D. Modern applied statistics with S. (Springer-Verlag, 2002).

38 Johnson, J. B. \& Omland, K. S. Model selection in ecology and evolution. Trends in Ecology \& Evolution 19, 101-108, (2004).

39 R Development Core Team. A language and environment for statistical computing ( $\mathrm{R}$ Foundation for Statistical Computing, Vienna, Austria, 2012). 
649

Figure legends:

Figure 1. Local species richness ( $\bar{\alpha}$ diversity) and community dissimilarity ( $\beta$ diversity) interact to affect average multifunctionality. a, average number of species per plot within spatial blocks ( $\bar{\alpha}$ diversity); b, dissimilarity in species composition among plots within spatial blocks ( $\beta$ diversity). The average level of multiple functions increased with $\bar{\alpha}$ diversity at intermediate to high (Int-High) $\beta$ diversity (slope and 95\% CI on the $\log \bar{\alpha}$ scale $=0.05(0.021-$ $0.086)$ ), and with $\beta$ diversity at high (High) $\bar{\alpha}$ diversity $(0.10(0.015-0.23))$, but was unrelated to $\bar{\alpha}$ diversity at low (Low) $\beta$ diversity $(-0.011(-0.057-0.034)$ and to $\beta$ diversity at low to intermediate (Low-Int) $\bar{\alpha}$ diversity $(-0.0044(-0.051-0.059)$.

Figure 2. Simulating reduced ecological interactions between local communities did not influence the relationships of plant diversity with average multifunctionality. Standardized regression coefficients of local species richness $(\bar{\alpha})$ and community dissimilarity $(\beta)$ with average multifunctionality for $\mathbf{a}$ and $\mathbf{b}$, observed landscapes (spatial blocks) composed of interconnected local plots within site (a) or within habitat (b), c and d, artificially constructed landscapes simulating reduced interconnection between local communities within sites (c) or within habitat (d). Standardized regression coefficients are shown with their 95\% confidence intervals such that diversity effect on multifunctionality is significant when the intervals do not overlap zero.

Figure 3. Relationships between the proportion of species maintaining ecosystem functioning and the number of ecosystem functions (a) or the number of spatial blocks (b) 

multimodel inference $0.247(0.239-0.256))$ or $\mathbf{b}$, more spatial blocks (slopes and 95\% CI: stepwise-deletion $0.252(0.233-0.271)$, randomization tests $0.387(0.364-0.410)$, multimodel 668 inference $0.381(0.345-0.418))$. Regression lines indicate generalized linear model fit for each 669 method with $95 \%$ confidence intervals. $\mathrm{N}$ denotes the number of sites included in each approach. 


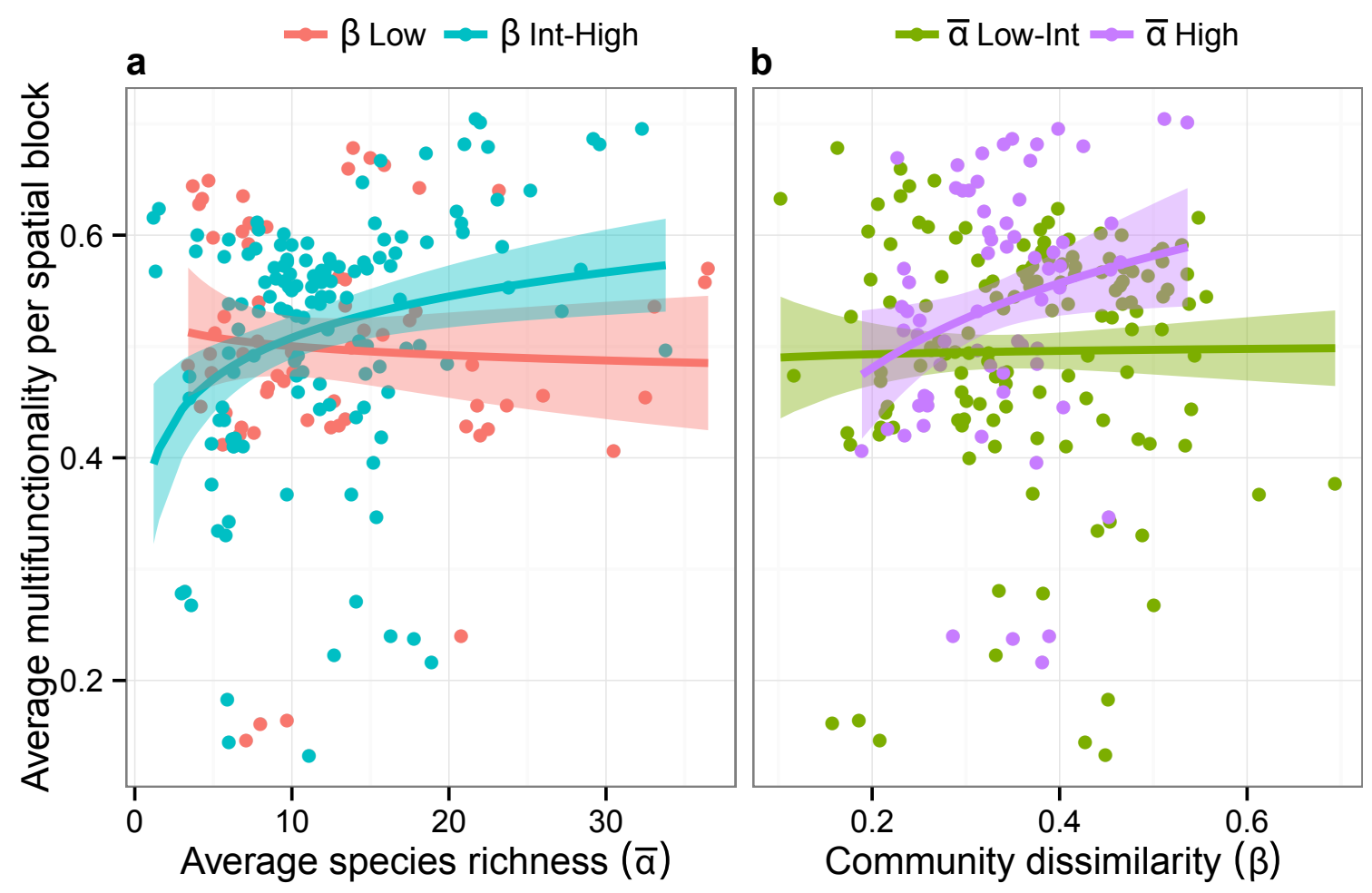


Observed landscapes

a (spatial blocks within site)

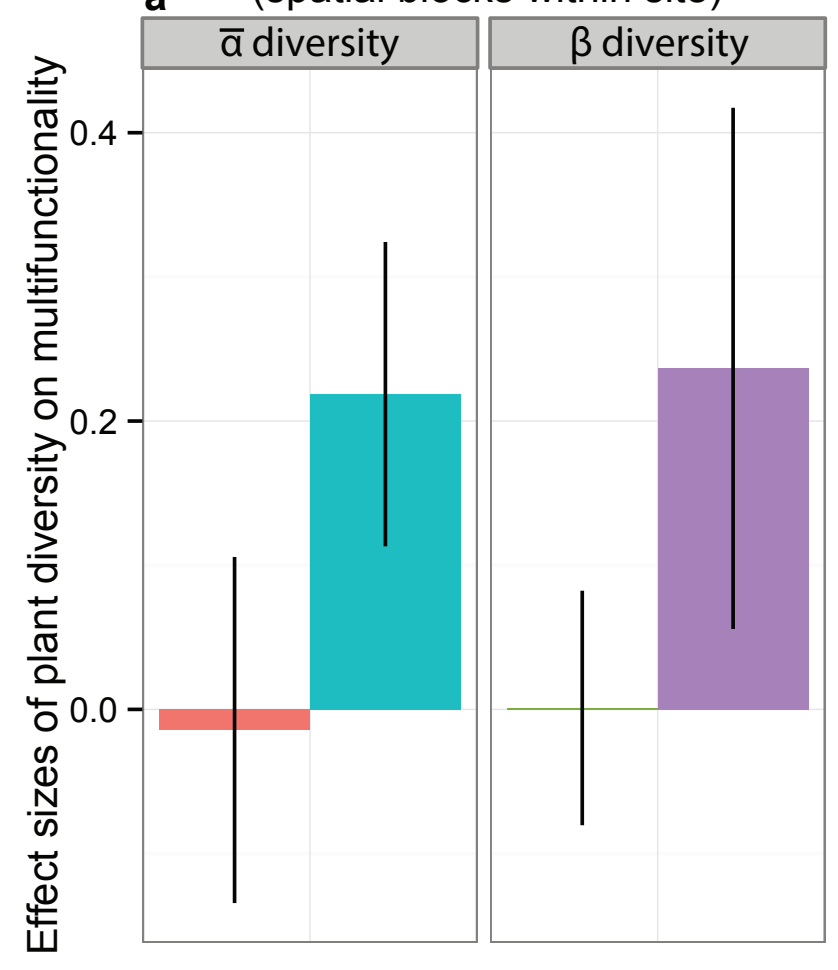

$\beta$ diversity $\bar{a}$ diversity

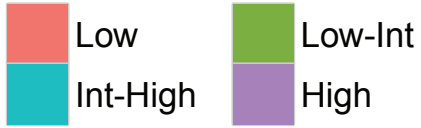

Simulated landscapes

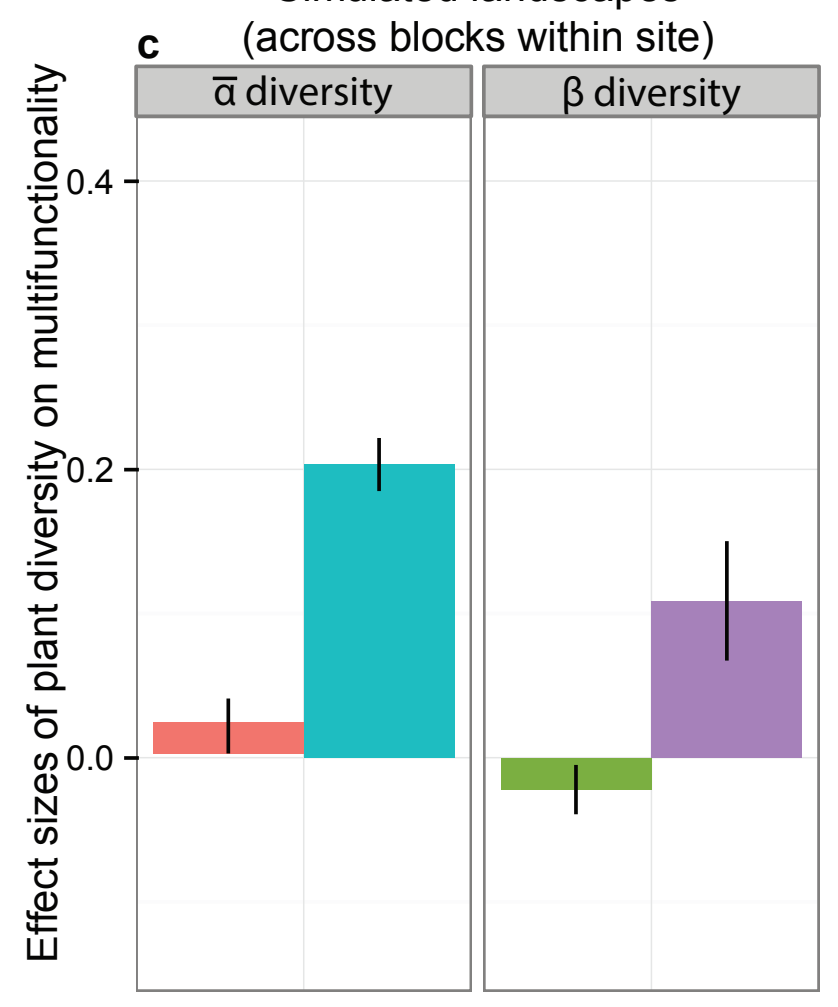

\section{b}

bserved landscapes

(spatial blocks within habitat)

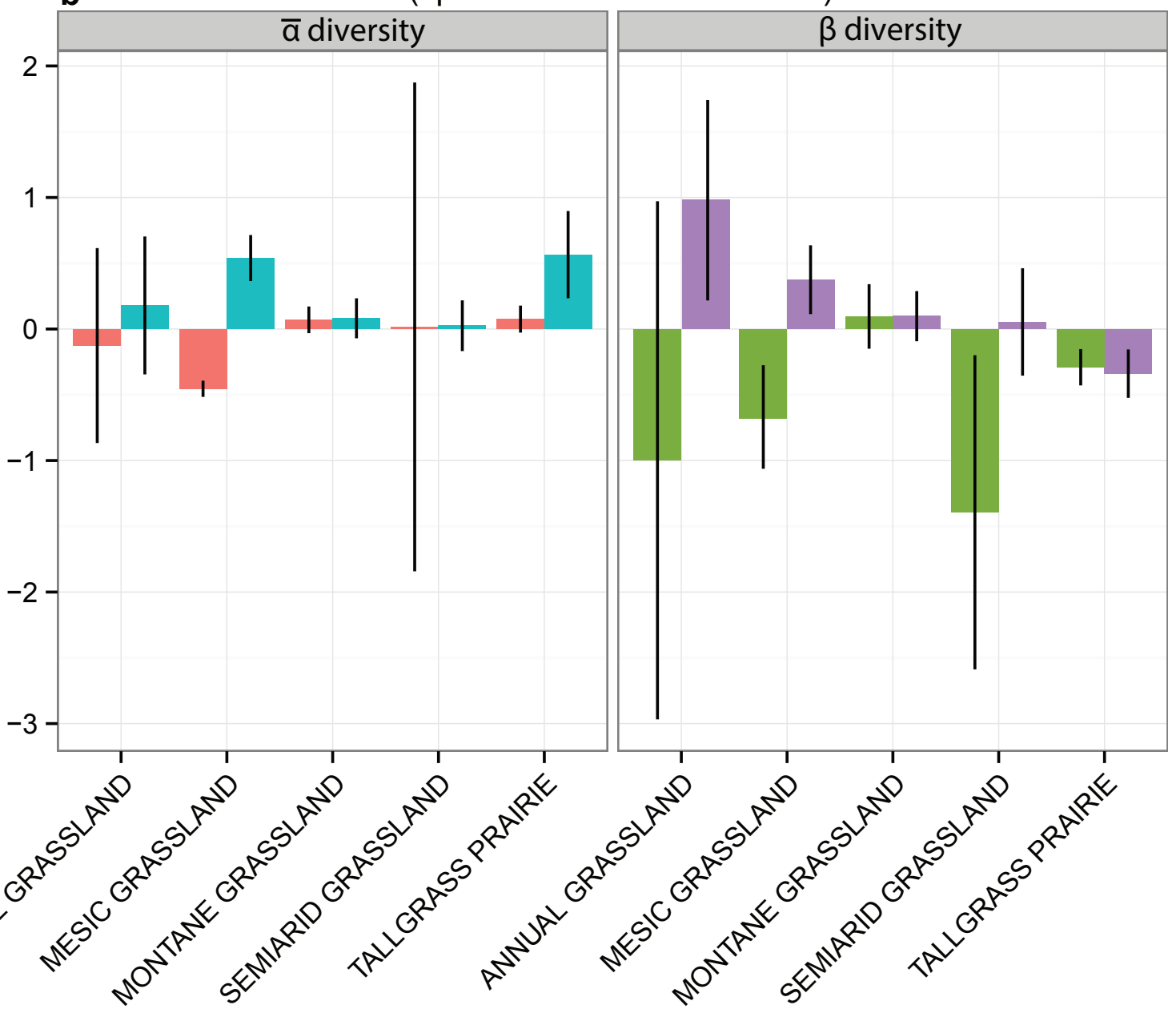

Simulated landscapes

(across sites within habitat)

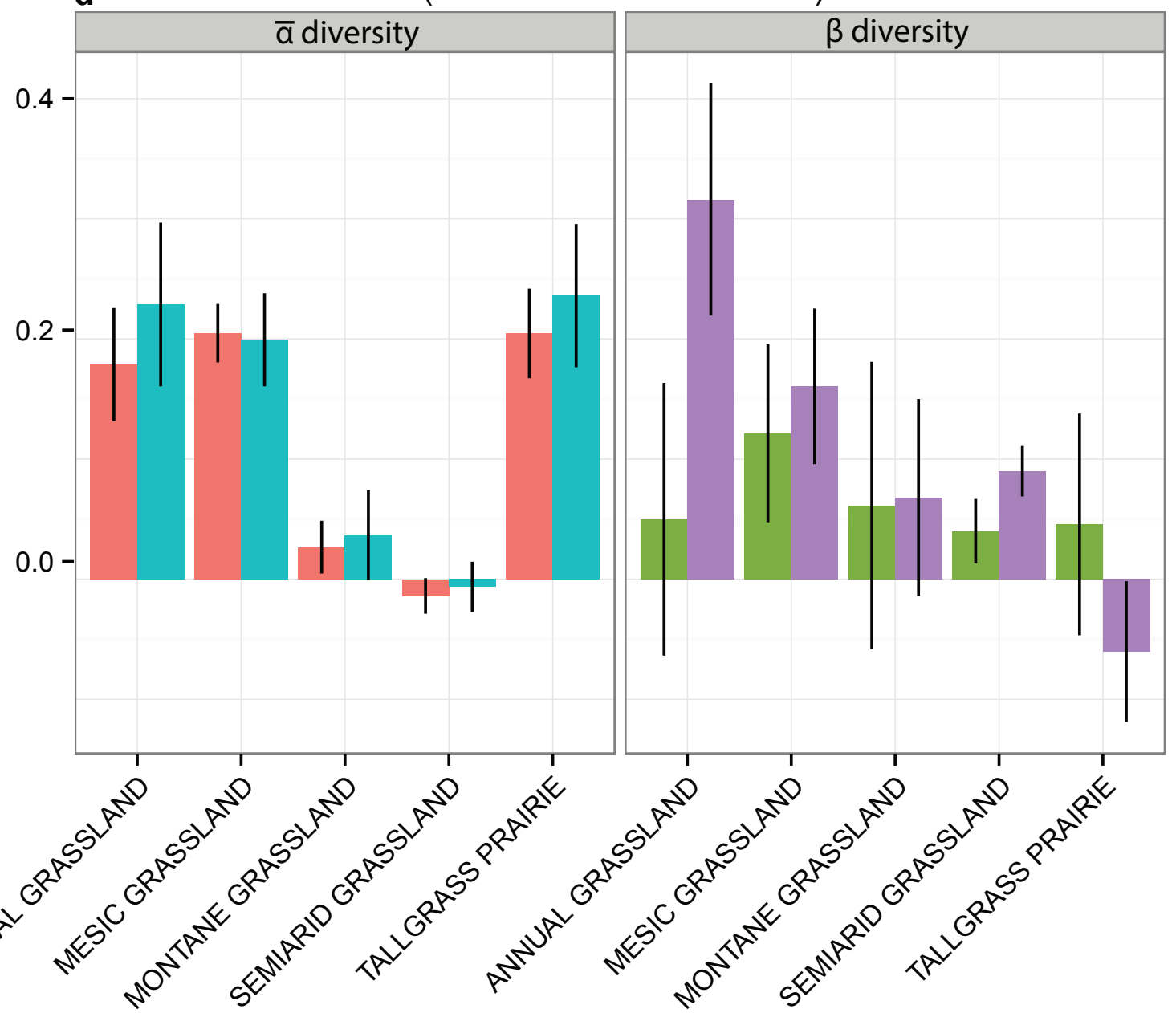


a

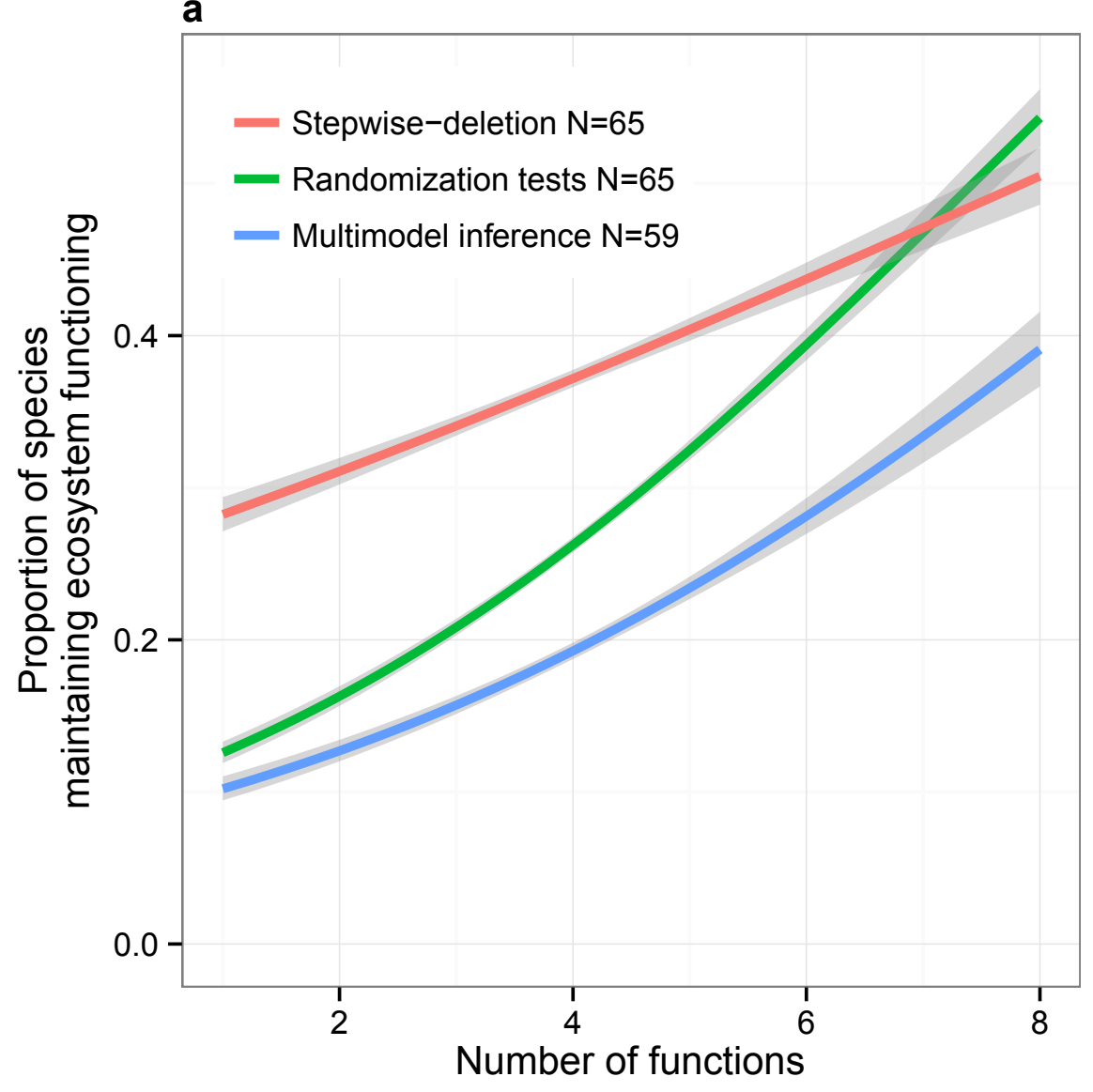

b

- $\mathrm{N}=62$

- $\mathrm{N}=62$

$\mathrm{N}=53$

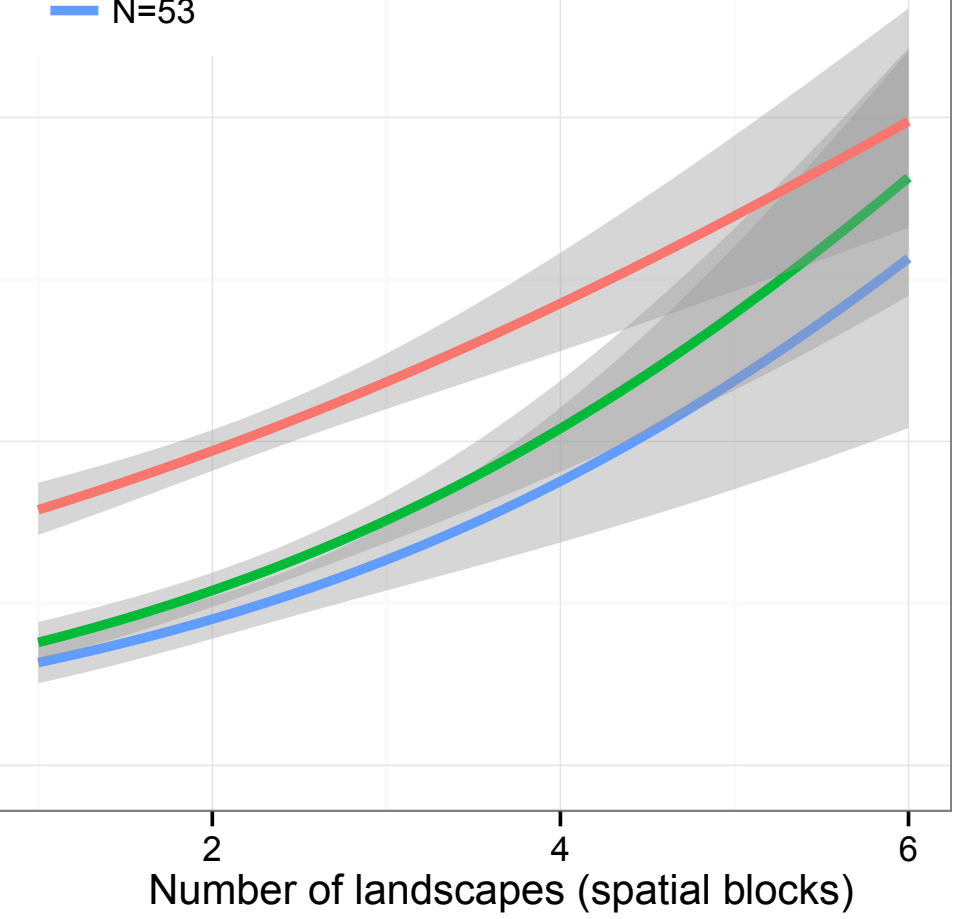

